\title{
Avaliação da velocidade de onda de pulso como marcador de rigidez arterial na artrite reumatoide: uma revisão integrativa
}

Evaluation of the pulse wave velocity as

marker of arterial rigidity in the artrite

FisiSenectus . Unochapecó Ano 7, n. 1 - Jan/Jun. 2019 reumatoide: an integrative review

\section{Priscila Roberta Reck. reckpri@gmail.com}

Fisioterapeuta. Mestranda no Programa de Pós-Graduação em Ciências do Movimento Humano na Universidade do Estado de Santa Catarina (CEFID/UDESC).

\section{Melissa Andrea Jeannet Michaelsen Cardoso Mezzari. andreajeannet@gmail.com}

Fisioterapeuta. Mestre e Doutoranda no Programa de Pós-Graduação em Ciências do Movimento Humano na Universidade do Estado de Santa Catarina (CEFID/UDESC).

Juliane de Oliveira. julifisioterapia@gmail.com

Fisioterapeuta. Doutoranda no Programa de Pós-Graduação em Ciências do Movimento Humano na Universidade do Estado de Santa Catarina (CEFID/UDESC).

\section{Juliana Gomes Pinheiro Cavalcante Rodrigues. juliana-adsj@hotmail.com}

Fisioterapeuta. Mestranda no Programa de Pós-Graduação em Ciências do Movimento Humano na Universidade do Estado de Santa Catarina (CEFID/UDESC).

Susana Cristina Domenech. scdomenech@gmail.com

Doutora em Engenharia de Materiais pela Universidade Federal de Santa Catarina (UFSC) - Doutorado sanduíche (CNPq) pela Martin-Luther Universität Halle-Wittenberg (Alemanha) e Albert-Ludwigs Universität Freiburg (Alemanha). Mestre em Engenharia de Minas, Metalúrgica e de Materiais pela Universidade Federal do Rio Grande do Sul (UFRGS). Licenciada e bacharel em Química. Professora do Programa de Pós-Graduação em Ciências do Movimento Humano da Universidade do Estado de Santa Catarina (CEFID/UDESC).

\section{Resumo}

Introdução: a rigidez arterial tem se mostrado um parâmetro importante na avaliação do risco cardiovascular. A medida da velocidade de onda de pulso arterial (VOP), por ser de fácil aplicabilidade e de amplo corpo de evidências, é considerada atualmente o padrão para avaliar a rigidez das artérias. Pacientes com artrite reumatoide (AR) apresentam um maior risco a doenças cardiovasculares (DCV) quando comparados à população em geral. As DCV são responsáveis pelo alto índice de morbimortalidade. Objetivo: esta revisão integrativa teve como objetivo, identificar quais os métodos mais utilizados na atualidade para avaliação da VOP em pacientes com AR. Método: trata-se de uma revisão integrativa, a partir de buscas em bases de dados da área da saúde como Bireme, Science Direct e PubMed. A estratégia de busca detalhada utilizou descritores em inglês e português, com os seguintes termos e operadores: "Pulse Wave Velocity" AND "Rheumatoid Arthritis" AND "Cardiovascular Risk"; Velocidade de onda de pulso arterial AND "Artrite Reumatoide" AND "Risco cardiovascular". Resultados: nesta revisão integrativa encontraram-se 125 artigos, pré-selecionados

\section{Fisißenectus}


pela pesquisa e pelos filtros bem como três estudos na revisão das referências. Destes, 83 foram excluídos a partir da leitura dos títulos, e 29 artigos, depois da leitura dos resumos. Dessa forma, foram selecionados 16 artigos para leitura completa do texto. Conclusão: a partir desta revisão integrativa, concluiu-se que os diversos equipamentos para análise da VOP, bem como as diferentes formas de aplicação, apresentam confiabilidade, visto que os valores e resultados apresentados pelos estudos na população com AR foram em sua maioria similares.

\title{
Palavras-chave
}

Artrite Reumatoide; Velocidade de Onda de Pulso Arterial; Avaliação.

\begin{abstract}
Introduction: The arterial rigidity has if shown an important parameter in the evaluation of the cardiovascular risk. The measure of the speed of wave of arterial (PWV) pulse, for being of easy applicability and of wide body of evidences, it is considered the pattern today to evaluate the rigidity of the arteries. Patient with arthritis reumatoide (RA) they present a larger risk to cardiovascular (DCV) diseases when compared the population in general, those, responsible for the high morbimortalidade index Objective: This revision integrativa had as objective, to identify which the methods more used at the present time for evaluation of the PWV in patients with RA. Methods: It is a revision integrativa, starting from searches in bases of data of the area of the health as Bireme, Science Direct and PubMed. The strategy of detailed search used descritores in English and Portuguese, with the following ones we have and operators: it "Presses Wave Velocity" AND "Rheumatoid Arthritis" "Cardiovascular AND Risk"; Speed of wave of arterial pulse AND "Artrite Reumatoide" AND "cardiovascular Risk". Results: In this integrative review were 125 articles, pre-selected by the research and the filters, besides 3 studies in the revision of the references. Of these, 83 were excluded starting from the reading of the titles and 29 after the reading of the summaries. In that way, 16 articles were selected for reading completes of the text. Conclusion: Starting from this revision integrativa, it was ended that the several equipments for analysis of PWV, as well as the different application forms, present reliability, because the values and results presented by the studies in the population with RA, they were in his/her similar majority.
\end{abstract}

\section{Keywords}

Rheumatoid arthritis; Arterial Pulse Wave Velocity; Evaluation.

\section{Introdução}

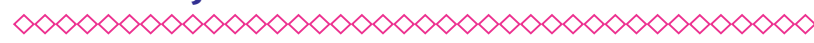

O sistema arterial é complexo e heterogêneo, com importantes diferenças entre as grandes e pequenas artérias, as arteríolas e a microcirculação. Sabe-se também que fatores genéticos e ambientais têm seu papel nas mudanças funcionais e estruturais da parede arterial, tendo como consequência a redução da elasticidade e aumento da rigidez arterial ${ }^{1}$. O envelhecimento vascular é consequência das mudanças que ocorrem nas propriedades biomecânicas da parede vascular, processo que pode ser acelerado pela hipertensão arterial (HAS), inflamações crônicas e comorbidades, como tabagismo, diabetes mellitus (DM) e dislipidemia².

A rigidez arterial tem se mostrado um parâmetro importante na avaliação do risco cardiovas- cular. A medida da Velocidade de Onda de Pulso (VOP), por ser de fácil aplicabilidade e de amplo corpo de evidências e relacionar-se com doenças cardíacas, é considerada atualmente o padrão ouro e um dos principais métodos para avaliar a rigidez das artérias e determinar a elasticidade de grandes vasos ${ }^{3}$. A VOP pode ser definida como sendo a distância percorrida pelo fluxo sanguíneo dividida pelo tempo que leva para percorrer uma distância total e, dessa forma, pode ser medida entre as artérias carótida, radial, femoral e distal ${ }^{4}$.

A Artrite Reumatoide (AR) é a doença autoimune mais frequente cuja prevalência mundial é estimada em 0,5 a $1 \%$ da população. A incidência anual dessa doença é de 40 novos casos a cada 100.000 mulheres e a metade desse valor em homens $^{5}$. Embora haja registro em todas as faixas etárias, há um predomínio em pacientes de 40 a 
60 anos, ${ }^{6}$ ou seja, durante a faixa etária de maior produtividade profissional.

Pacientes com AR apresentam um maior risco a doenças cardiovasculares (DCV) quando comparados à população em geral, sendo essas enfermidades responsáveis pelo alto índice de morbimortalidade. Estudos indicam que dentre os efeitos mais comuns da AR, está o infarto agudo do miocárdio, com prevalência de 1 a $17 \%$, e que eventos como isquemia coronariana e acidente vascular encefálico, representam um índice $50 \%$ maior em pacientes portadores de $\mathrm{AR}^{7}$. Além disso, o processo inflamatório da AR apresenta algumas similaridades com o componente inflamatório da aterosclerose, como níveis elevados de proteína C Reativa ( $P C R$ ), citocinas e fibrinogênio, que contribuem direta ou indiretamente na formação de placas ateroscleróticas ${ }^{8}$.

Considerando que doenças inflamatórias crônicas tendem a favorecer a rigidez arterial e a formação de placa ateriosclerótica e que a VOP é considerada o padrão ouro para avaliação, o presente estudo teve como objetivo identificar por meio de uma revisão integrativa, quais os métodos mais utilizados na atualidade para avaliação da VOP em pacientes com AR.

\section{Método}

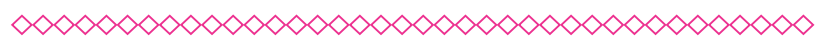

0 presente estudo consiste em uma revisão integrativa, na qual foram realizadas buscas em bases de dados voltadas à área da saúde.

Para realização desta revisão, foram seguidas duas etapas: a primeira foi referente à procura dos descritores estabelecidos pelo DeCS (Descritores em Ciências da Saúde) da Biblioteca Virtual em Saúde. Em seguida, foram estabelecidos dois critérios para filtro dos artigos: estudos realizados nos últimos dez anos e idiomas inglês ou português.

Como critérios de inclusão dos estudos, foram considerados artigos que avaliaram a rigidez arterial em pacientes com AR por meio de sistemas que determinam a VOP de forma não invasiva; testes clínicos randomizados e estudos observacionais (coorte prospectiva e retrospectiva e estudos caso controle). Para melhorar o filtro na busca dos artigos, foram estabelecidos alguns critérios de exclusão, como: utilização de outros métodos de avaliação da VOP que não fossem por tonometria ou oscilométricos; teses e dissertações, revisões sistemáticas, guidelines, resumos de congressos, editoriais e cartas dos autores, livros, notas/ comentários, artigo modelo animal, ou que não estivessem disponíveis na íntegra.

A busca foi efetuada em bases de Bireme, Science Direct e PubMed. Além disso, foram realizadas buscas na lista de referências dos estudos elegíveis e revisões sistemáticas anteriores. A estratégia de busca detalhada utilizou descritores em inglês e português, com os seguintes termos e operadores: "Pulse Wave Velocity" AND "Rheumatoid Arthritis" AND "Cardiovascular Risk"; "Velocidade de onda de pulso arterial" AND "Artrite Reumatoide" AND "Risco cardiovascular".

Para evitar a inclusão de artigos repetidos, os títulos dos artigos foram inseridos em uma planilha do Excel (Microsoft Office, 2010), excluindo referências semelhantes, obtendo-se assim, uma soma real quanto ao número de artigos referente ao tema.

A seleção dos estudos foi processada por dois avaliadores, simultaneamente e de forma independente, seguindo os critérios de inclusão pré-estabelecidos. Estudos que não aprestaram informações suficientes para exclusão pela leitura do título ou resumo, foram lidos na íntegra. Qualquer divergência quanto à seleção do conteúdo foi discutida até obter-se consenso entre os avaliadores. A extração dos dados foi realizada seguindo uma ficha padronizada com as seguintes informações: autor, ano de publicação, objetivo do estudo, sistema, método e protocolo de avaliação da VOP.

\section{Resultados}

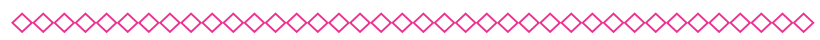

Nesta revisão integrativa encontraram-se 125 artigos, pré-selecionados pela pesquisa e pelos filtros bem como três estudos na revisão das referências. Dos quais, 83 foram excluídos a partir da leitura dos títulos, e 29 artigos, depois da leitura dos resumos. Dessa forma, foram selecionados 16 
artigos para leitura completa, que foram organizados e resumidos de forma padronizada (Quadro 1 ). Nesta revisão foram localizados quatro principais protocolos de avaliação da VOP, Complior ${ }^{\circledR}$ System, VP-2000/1000 - Colin Co Ltda, PulsePen ${ }^{\circledR}$, SphygmoCor-AtCor Medical.

\section{Discussão}

$\infty \times \infty \times \infty \times \infty \times \infty \times \infty \times \infty \times \infty \times \infty \times \infty \times \infty \times \infty \times \infty$

A rigidez arterial é considerada um parâmetro importante para avaliação de doenças e fatores de risco cardiovascular. Dentre os inúmeros métodos e formas de avaliação, destaca-se a medida da VOP, devido à relativa facilidade na determinação e confiabilidade percebida, sendo incluída na prática e no âmbito de estudos clínicos ${ }^{3}$.

Equipamentos que medem a pressão arterial de quatro membros e a VOP foram fabricados pela primeira vez no Japão e atualmente são populares em outros países asiáticos onde são utilizados principalmente para fins de pesquisa. Esses dispositivos são capazes de medir a VOP de artérias carótidas proximais e distais, índice de aumento (Aix) e pressão arterial central bem como outros índices similares ${ }^{9}$.

Dentre os aparelhos que medem a VOP de forma não invasiva, destacam-se os modelos oscilométricos, piezoelétricos e os tonométricos, que apresentam boa correlação com os métodos mais utilizados: o Sistema Complior ${ }^{\circledR}$ (França), ou o SchygmoCor ${ }^{\circledast}$ (Austrália). 0 autor observa que esses métodos se destacam pela fácil aplicabilidade, pouco tempo de treinamento do operador, otimização do tempo de aplicação e realização do exame, tornando-se mais útil e viável na prática clínica ${ }^{10}$.

A seguir, a descrição dos quatro principais protocolos de avaliação da VOP, que se destacaram na busca para esta revisão.

\section{1) Complior ${ }^{\circledR}$ System}

Desenvolvido na França, o Complior ${ }^{\circledR}$ System é um dispositivo automático que registra as ondas de pulso de forma contínua e calcula a VOP. Por meio do sistema, obtém-se uma medida mecanizada da VOP realizada a partir do posicionamento de transdutores mecânicos sobre a pele, possibilitando a avaliação de dois segmentos arteriais de forma simultânea, principalmente o tronco aórtico e os membros superiores e inferiores ${ }^{4}$.

Dentre as formas de aplicação do equipamento, destacam-se as medidas da VOP carótida-femoral (CF), sendo esse o único método reconhecido como medida de rigidez aórtica pelas Sociedades Europeias de Hipertensão e Cardiologia (ESH-ESC) ${ }^{3}$. O cálculo da VOP-CF é realizado pela distância da medida entre os transdutores posicionados na artéria proximal (artéria carótida) e na distal (artéria femoral), dividido pelo tempo de atraso de registro entre as ondas 4 .

Dos estudos encontrados, dois utilizaram somente o equipamento Complior $^{\circledR}$ System como método de avaliação da VOP e ambos utilizaram as medidas CF como base na aplicação ${ }^{11,12}$. Entretanto, somente um trabalho especificou o protocolo utilizado durante a avaliação ${ }^{12}$ que foi realizada com transdutores posicionados em membro superior direito depois do paciente permanecer em repouso por 15 minutos. Um terceiro estudo utilizou o Complior ${ }^{\circledR}$ System para avaliar a VOP das artérias CF e um segundo equipamento, o SphygmoCor - AtCor Medical, para mensurar o índice de aumento (Aix) da forma da onda da pressão central aórtica $(\text { CPAM) })^{13}$.

\section{2) VP-2000/1000 - Colin Co Ltda}

0 equipamento VP-2000/1000 foi desenvolvido pela Omrom Healthcare Co. Ltd., no Japão, destinado a profissionais da área da saúde com o intuito de auxiliar no diagnóstico de doenças vasculares periféricas. 0 dispositivo permite calcular clinicamente índices das artérias braquial e do tornozelo, pressão arterial, VOP e o Aix, obtendo-se variáveis com auxílio de manguitos oscilométricos adaptados para registros pletismográficos ${ }^{14}$.

O VP-2000/1000 obtém de forma não invasiva, medidas referentes à pressão arterial, frequência cardíaca, ECG, formas de onda de pulso e som cardíaco ${ }^{14}$. Para determinar a VOP, calcula-se o tempo de trânsito entre as ondas dividindo-o pela distância percorrida.

Um estudo realizado na China investigou a relação da VOP com fatores de riscos de pacientes com AR. A avaliação da VOP foi realizada de modo 
não invasivo pelo equipamento VP-2000 Colin Co. Ltd., Komaki, Japão. Para realização dessa verificação, os indivíduos foram orientados a permanecerem cinco minutos em repouso e os sensores foram posicionados na região da artéria do punho e tornozelo de ambos hemicorpos. Os resultados foram classificados em VOP alto e baixo, e demonstraram que a idade mais avançada e perfil lipídico elevado, principalmente triglicerídeos, podem ser os principais determinantes da rigidez arterial de pacientes chineses com $\mathrm{AR}^{15}$.

Para analisar a prevalência de doença vascular e investigar fatores de riscos cardiovasculares relacionados à AR que influenciam a VOP além dos riscos tradicionais, Kim et al. ${ }^{16}$ avaliaram 262 pacientes coreanos diagnosticados com AR. A aplicação e execução da avaliação da VOP seguiram os mesmos passos e parâmetros citados no estudo de Li et al. ${ }^{15}$, utilizando-se as medidas da VOP do membro superior direito (MSD) e membro superior esquerdo (MSE), porém, além da VOP, foi avaliado também o Aix.

\section{3) PulsePen ${ }^{\circledR}$}

Desenvolvido pela DiaTecne, na Itália, o dispositivo PulsePen ${ }^{\circledR}$ tem como base o princípio de tonometria de aplanação, ou seja, o sensor é posicionado sobre a pele onde localiza-se a artéria a ser avaliada (radial, femoral e carótida) e a partir disso, realiza-se a medida da VOP e estima-se os reflexos das ondas por meio da análise das formas de onda da PA obtidas ${ }^{17}$.

Esse equipamento é composto de uma sonda tonométrica e de uma unidade de ECG que estima automaticamente a VOP. Como forma de aplicação, preconiza-se que o avaliador posicione 0 sensor do tonômetro na artéria carótida comum, enquanto realiza de forma simultânea o ECG. Tanto a pressão quanto os sinais eletrocardiográficos são transmitidos para um computador e a análise realizada por um software especialmente projetado ${ }^{17}$.

0 instrumento foi utilizado no estudo de Mercado et al. ${ }^{2}$, com o objetivo de avaliar o impacto do tempo da doença na VOP da carótida para a femoral em pacientes com AR, sem conhecer fatores de riscos tradicionais ou comorbidades prévias. Como protocolo de aplicação, os autores relataram que a coleta da VOP ocorreu em dois intervalos, sendo o primeiro com o tonômetro posicionado na artéria carótida, simultâneo a um ECG e posteriormente repetindo o procedimento na artéria femoral. O cálculo da VOP foi determinado pela distância entre os dois pontos avaliados, dividido pelo tempo de atraso entre os pulsos detectados. 0 estudo não especifica qual o posicionamento do paciente durante a avaliação bem como se houve tempo de repouso e qual o segmento avaliado (hemicorpo direito ou esquerdo).

\section{4) SphygmoCor - AtCor Medical}

Disponível comercialmente há mais de uma década, o SphygmoCor foi desenvolvido pela AtCor Medical, Sydney/Austrália e consiste em um instrumento que avalia a rigidez arterial por meio da tonometria de aplanação que tem sido extensivamente usada, é de fácil aplicabilidade e reprodutibilidade razoável ${ }^{18}$.

Para se obter a VOP, a onda de pulso é registrada de forma sequencial na artéria carótida e artéria femoral pelo transdutor, simultâneo a um ECG utilizado como referência para calcular o tempo de trânsito. 0 autor afirma que, com o equipamento, também é possível registrar a forma de onda de pulso radial, e a partir da tonometria de aplanação, emprega-se uma transferência generalizada para se obter a forma da onda da pressão aórtica central, e depois com a calibração da pressão arterial braquial e a suposição da pressão arterial média constante sobre as grandes artérias derivadas da pressão arterial sistólica central e da PP18.

Nesta revisão integrativa, houve predomínio por estudos que utilizaram o SphygmoCor como instrumento de avaliação da VOP. Destes, seis investigaram a ação de medicamentos utilizados no tratamento da AR sobre a rigidez arterial e o risco a DCV desses pacientes ${ }^{19,20,21,22,23,24}$, dois artigos estudaram o impacto da AR sobre a rigidez arterial ${ }^{25,26,27}$; e Pieringer et al. ${ }^{28}$ investigou a associação entre dois conceitos para avaliação vascular em pacientes com AR, o SCORE e o QRISK2.

Apenas os estudos de Mathieu et al. ${ }^{22}$, de MäkiPetäjä et al. ${ }^{23}$ e de Wong et al. ${ }^{24}$ relataram parcialmente qual o protocolo e o método de avaliação utilizado na obtenção da VOP, considerando tempo de repouso e artérias que foram avaliadas. Os 
demais estudos não especificaram qual o protocolo e o método empregado na avaliação da rigidez arterial por meio da VOP19,20,21,25,28.

Em relação aos equipamentos utilizados para avaliação da rigidez arterial, percebeu-se que a maioria tem como referência para obtenção da VOP, medidas das artérias carótida e femoral, e um deles apenas, baseia-se nas medidas de artérias braquial e tornozelo. Outra questão importante referente aos instrumentos, é que os artigos encontrados apresentam apenas modelos que utilizam na avaliação, o princípio da tonometria ou a oscilometria.

Sobre a descrição do método de avaliação, poucos estudos encontrados preocuparam-se em relatar de forma detalhada quais os procedimentos adotados na obtenção da VOP em relação ao paciente e ao equipamento, o que por sua vez prejudica a reprodutibilidade das técnicas e medidas da rigidez arterial. A descrição correta e detalhada de protocolos de avaliações é de extrema importância, entendendo que a padronização de métodos, favorece a confiabilidade dos estudos.

\section{Conclusão}

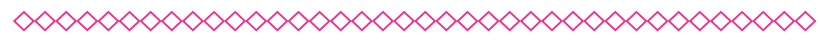

A partir da revisão integrativa, concluiu-se que os equipamentos para análise da VOP, bem como diferentes formas de aplicação, apresentam confiabilidade para avaliação da rigidez arterial, destinada à investigação de diferentes desfechos relacionados ao risco de DCV, em especial, em indivíduos com AR.

Diversos equipamentos, métodos e procedimentos são propostos em estudos para avaliação da VOP, de forma não invasiva. Quando comparados, encontraram-se alguns diferentes modelos, como os oscilométricos, piezoelétricos e os tonométricos, com boa correlação com os métodos mais utilizados: o Sistema Complior ${ }^{\circledR}$ (França), ou o SchygmoCor ${ }^{\circledR}$ (Austrália). Esses métodos destacam-se pela fácil aplicabilidade, pouco tempo de treinamento do operador, otimização do tempo de aplicação e realização do exame, tornando-se mais útil e viável na prática clínica.

\section{Agradecimentos}

$\infty<\infty<\infty<\infty<\infty<\infty<\infty<\infty<\infty<\infty<\infty<\infty<\infty<\infty<\infty<\infty<\infty$

0 presente estudo foi realizado em conjunto com o Laboratório de Instrumentação - (LABIN) e Laboratório de Análises Multissetorial (MULTILAB) da Universidade do Estado de Santa Catarina (UDESC), os quais contam com recursos disponibilizados pela FAPESC, CNPq e CAPES, para desenvolvimento e execução de diversos projetos vinculados a este artigo.

\section{Referências}

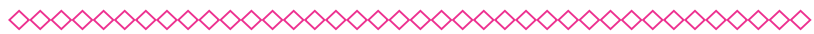

1. Favero FF. Avaliação da rigidez arterial pelo método da velocidade de onda de pulso (VOP) em hipertensos resistentes e controlados. [Dissertação]. São Paulo: Universidade Estadual de Campinas, Faculdade de Ciências Médicas; 2009

2. Mercado MV, Bañuelos EG, MD, Avila EC, Muñoz EC, Becerra CB, Sanchez AA, et al. Disease duration of rheumatoid arthritis is a predictor of vascular stiffness: a cross-sectional study in patients without known cardiovascular comorbidities: A STROBE-compliant article. Medicine. 2017;96(33):e7862.

3. Mancia G, Backer G, Dominiczak A, Cifkova R, Fagard R, Guido GG, et al. Guidelines for the management of arterial hypertension: The Task Force for the Management of Arterial Hypertension of the European Society of Hypertension (ESH) and of the European Society of Cardiology (ESC). European Heart J. 2007;28(12):1462-536.

4. Laurent S, Cockcrofrt J, Bortel LV, Boutouyrie P, Giannattasio C, Hayoz D. et al. Expert consensus document on arterial stiffness: methodological issues and clinical application. European Heart J. 2006;27(21):2588-605.

5. Brasil. Ministério da Saúde [Internet]. Portal da Saúde - Ministério da Saúde. [acesso em 19 mar 
2019]. Disponível em: http://portal.saude.gov. br/404.html

6. Mota LMHD, Cruz BA, Brenol CV, Pereira IA, Rezende-Fronza LS, Bertolo MB, et al., Diretrizes para o diagnóstico da artrite reumatoide. Rev Bras de Reumatologia. 2013;53(2):141-57.

7. Gomes RKS, Albers AC, Salussoglia AIP, Bazzan AM, Schreiner LC, Vieira MI, et al. Prevalência de doença isquêmica cardíaca e fatores associados em pacientes com artrite reumatoide no Sul do Brasil. Rev Bras de Reumatologia. 2016;57(5): 412-18.

8. Brenol CV, Monticielo OA, Xavier RM, Brenol JCT, Artrite reumatóide e aterosclerose. Rev Assoc Med Bras. 2007;53(5):465-70.

9. Ato D. Pitfalls in the ankle-brachial index and brachial-ankle pulse wave velocity. Vascular health and risk management. 2018;14:41-62.

10. Ring M, Eriksson MJ, Zierath JR, Caidahl K. Arterial stiffness estimation in healthy subjects: a validation of oscillometric (Arteriograph) and tonometric (SphygmoCor) techniques. Hypertension Research. 2014;37(11):999-1007.

11. Botta E, Meroño T, Saucedo C, Martín M, Tetzlaff W, Sorroche P, Boero L. et al. Associations between disease activity, markers of HDL functionality and arterial stiffness in patients with rheumatoid arthritis. Atherosclerosis. 2016;251:438-44.

12. Magda SL, Mincu RI, Florescy M, Ciobanu AO, Udrea GF, Cinteza M. et al. The assessment of subclinical cardiovascular dysfunction in treated rheumatoid artritis. Maedica. 2016;11(4):267-76.

13. Vassilopoulos D, Gravos A, Vlachopoulos C, Kandili A, Loakeimidis N, Pectasides D. et al. Adalimumab decreases aortic stiffness independently of its effect in disease activity in patients with rheumatoid arthritis. Clinical Rheumatology. 2015;34(2):359-64.

14. Suzuki E, Kashiwagi A, Nishio Y, Egwa K, Shimizu S, Maegawa $\mathrm{H}$. et al. Increased arterial wall stiffness limits flow volume in the lower extremities in type 2 diabetic patients. Diabetes Care. 2001;24(12):2107-14.

15. Li P, Han C, Ma C, Guo J, Liu B, Du J. et al. Determinants of brachial-ankle pulse wave velocity in Chinese patients with rheumatoid arthritis. Clinical and Developmental Immunology. 2013;64(5):1124-30.

16. Kim YS, Sung YK, Choi CB, Uhm WS, Kim TH, Shi JH. et al. The major determinants of arterial stiffness in Korean patients with rheumatoid arthritis are age and systolic blood pressure, not disease-related factors. Rheumatology international. 2012;32(11):3455-61.

17. Salvi P, Lio L, Labat C, Ricci E, Pannier B, Benetos A. Validation of a new non-invasive portable tonometer for determining arterial pressure wave and pulse wave velocity: the PulsePen device. J of hypertension. 2004;22(12):2285-93.

18. Frimodt MM, Nielsen AH, Kamper Al, Strandgaard S. Reproducibility of pulse-wave analysis and pulse-wave velocity determination in chronic kidney disease. Nephrol Dial Transplant. 2008;23(2):594-600

19. Mangoni A A, Baghdadi LR, Shanahan EM, Wiese MD, Tommasi S, Elliot D. et al. Methotrexate, blood pressure and markers of arterial function in patients with rheumatoid arthritis: a repeated cross-sectional study. Therapeutic advances in musculoskeletal disease. 2017;9(9):213-29.

20. Provan SA, Berg IJ, Hammer HB, Mathiessen A, Kvin TK,Semb AG. The impact of newer biological disease modifying anti-rheumatic drugs on cardiovascular risk factors: a 12-month longitudinal study in rheumatoid arthritis patients treated with rituximab, abatacept and tociliziumab. PloS one. 2015;10(6):e0130709.

21. Mcinnes $L$, Thompson L, Giles JT, Bathon $J M$, Salmon JE, Beaulieu AD. et al. Effect of interleukin-6 receptor blockade on surrogates of vascular risk in rheumatoid arthritis: MEASURE, a randomised, placebo-controlled study. Annals of the rheumatic diseases. 2015;74(4):694-702. 
22. Mathieu S, Pereira B, Dubost JJ, Lusson JR. No significant change in arterial stiffness in RA after 6 months and 1 year of rituximab treatment. Rheumatology. 2012; 51(6):1107-11.

23. Mäki-Petäjä K, Elkhawad M, Cheriyan J, Joshi FR, Östör AJK, Hall SC, et al. Anti-tumor necrosis factor- therapy reduces aortic inflammation and stiffness in patients with rheumatoid arthritis. Circulation. 2012;126:2473-80.

24. Wong M, Oakley SP, Young L, Jiang BY, Wierzbicki A, Panay G. et al. Infliximab improves vascular stiffness in patients with rheumatoid arthritis. Annals of the rheumatic diseases. 2009;68(8):1277-84.

25. Arida A, Zampeli E, Konstantonis G, Fragiadaki $K$, Kitas GD, Protogerou AD, et al. Rheumatoid arthritis is sufficient to cause atheromatosis but not arterial stiffness or hypertrophy in the absence of classical cardiovascular risk factors. Clinical Rheumatology. 2015;34(5):853-59.
26. Pieringer $H$, Brummaier T, Schimid M, Mag DI, Pichler M, Khayyati A, et al. Rheumatoid arthritis is an independent risk factor for an increased augmentation index regardless of the coexistence of traditional cardiovascular risk factors. In: Seminars in arthritis and rheumatism. WB Saunders. 2012;17-22.

27. Pieringer $H$, Stuby $U$, Pohanka E, Biesenbach $G$. Arterial stiffness in a muscular artery in women with longstanding rheumatoid arthritis compared with healthy controls and patients with traditional cardiovascular risk factors. Rheumatology international. 2010;30(10):1335-39.

28. Pieringer $H$, Pohanka $E$, Puchner $R$, Brummaier T. Associação entre a função vascular e o risco cardiovascular estimado em pacientes com artrite reumatoide. Rev Bras de Reumatologia. 2017;57(5):452-60. 


\section{Anexos}

Quadro 1 - Métodos e protocolos de avaliações da VOP em pacientes com AR

\begin{tabular}{|c|c|c|c|}
\hline $\begin{array}{l}\text { Autor e ano de } \\
\text { publicação }\end{array}$ & Objetivo & Sistema VOP & Método avaliação VOP \\
\hline $\begin{array}{l}\text { Vassilopoulos } \\
\text { et al. (2014) }\end{array}$ & $\begin{array}{l}\text { Investigar o efeito de } \\
\text { adalimumabe (ADA) na } \\
\text { rigidez arterial em pacientes } \\
\text { com AR, de acordo com } \\
\text { seu efeito na atividade da } \\
\text { doença. }\end{array}$ & $\begin{array}{l}\text { SphygmoCor, } \\
\text { AtCor Medica/ } \\
\text { Complior }{ }^{\circledR} \\
\text { Colson AS, } \\
\text { Paris, France }\end{array}$ & $\begin{array}{l}\text { Avaliação das artérias } \\
\text { carótida e femoral; } \\
\text { Não especificado protocolo } \\
\text { de avaliação. }\end{array}$ \\
\hline $\begin{array}{l}\text { Mäki-Petäjä et } \\
\text { al. (2012) }\end{array}$ & $\begin{array}{l}\text { Pacientes com AR } \\
\text { apresentam inflamação } \\
\text { aórtica, que pode ser } \\
\text { revertida com anti-TNF } \\
\text { (fator de necrose tumoral), } \\
\text { correlacionando-se com a } \\
\text { redução da rigidez aórtica. }\end{array}$ & $\begin{array}{l}\text { Sphygmocor- } \\
\text { AtCor Medical }\end{array}$ & $\begin{array}{l}\text { Avaliação carotida-braquial e } \\
\text { carótida femoral; } \\
\text { Paciente em posição supino } \\
\text { e repouso de } 15 \text { minutos; } \\
\text { Realizadas a média de duas } \\
\text { medidas. }\end{array}$ \\
\hline Li et al. (2013) & $\begin{array}{l}\text { Investigar a relação entre } \\
\text { a rigidez arterial e fatores } \\
\text { de risco associados em } \\
\text { pacientes chineses com AR. }\end{array}$ & $\begin{array}{l}\text { VP-2000, } \\
\text { Colin Co Ltd, } \\
\text { Komaki, Japão }\end{array}$ & $\begin{array}{l}\text { Avaliação carótida braquial e } \\
\text { carótida- distal; } \\
\text { Paciente em posição supina } \\
\text { e repouso por mais de } 5 \\
\text { minutos; } \\
\text { Medidas realizadas em } \\
\text { hemicorpos direito e } \\
\text { esquerdo. }\end{array}$ \\
\hline $\begin{array}{l}\text { Mercado et al. } \\
\text { (2017) }\end{array}$ & $\begin{array}{l}\text { Analisar o impacto do } \\
\text { tempo da doença na } \\
\text { velocidade da onda de pulso } \\
\text { da carótida-femoral (VOP- } \\
\text { CF) em pacientes com AR } \\
\text { sem qualquer fator de risco } \\
\text { cardiovascular tradicional } \\
\text { conhecido ou comorbidades } \\
\text { prévias. }\end{array}$ & $\begin{array}{l}\text { Pulse Pen } \\
\text { (DiaTecne s.r.l. } \\
\text { Milão, Itália) }\end{array}$ & $\begin{array}{l}\text { Avaliação individual das } \\
\text { artérias carotida e femoral; } \\
\text { Simultânea ao } \\
\text { Eletrocardiogama (ECG); } \\
\text { Não especificado protocolo } \\
\text { de avaliação. }\end{array}$ \\
\hline $\begin{array}{l}\text { McInnes et al. } \\
\text { (2015) }\end{array}$ & $\begin{array}{l}\text { Caracterizar o impacto } \\
\text { da inibição da IL-6R e } \\
\text { estabelecer os novos fatores } \\
\text { de risco na AR ativa. }\end{array}$ & $\begin{array}{l}\text { SphygmoCor } \\
\text { - AtCor, } \\
\text { San Jose, } \\
\text { Califórnia, EUA }\end{array}$ & $\begin{array}{l}\text { Não especificado protocolo } \\
\text { de avaliação. }\end{array}$ \\
\hline $\begin{array}{l}\text { Wong et al. } \\
\text { (2009) }\end{array}$ & $\begin{array}{l}\text { Avaliar os efeitos do } \\
\text { tratamento com infliximabe } \\
\text { na rigidez vascular e } \\
\text { estrutura em pacientes } \\
\text { com AR. }\end{array}$ & $\begin{array}{l}\text { SphygmoCor, } \\
\text { PWA Medical, } \\
\text { Sydney, } \\
\text { Austrália }\end{array}$ & $\begin{array}{l}\text { Avaliação da carótida } \\
\text { femoral e da artéria radial; } \\
\text { Paciente em jejum e em } \\
\text { posição supina. }\end{array}$ \\
\hline
\end{tabular}




\begin{tabular}{|c|c|c|c|}
\hline $\begin{array}{l}\text { Autor e ano de } \\
\text { publicação }\end{array}$ & Objetivo & Sistema VOP & Método avaliação VOP \\
\hline $\begin{array}{l}\text { Provan et al. } \\
\text { (2015) }\end{array}$ & $\begin{array}{l}\text { Avaliar se o tratamento com } \\
\text { um dos DMARD's biológicos: } \\
\text { rituximab, abatacept ou } \\
\text { tocilizumab reduzem os } \\
\text { fatores de risco de doença } \\
\text { cardiovascular (DCV) em } \\
\text { pacientes com AR. }\end{array}$ & $\begin{array}{l}\text { Sphygmocor } \\
\text { - Atcor, West } \\
\text { Ryde, Austrália }\end{array}$ & $\begin{array}{l}\text { Não especificado protocolo } \\
\text { de avaliação. }\end{array}$ \\
\hline $\begin{array}{l}\text { Arida et al. } \\
(2015)\end{array}$ & $\begin{array}{l}\text { Confirmar a hipótese } \\
\text { de que a AR por si só, } \\
\text { afeta diferencialmente } \\
\text { ateromatose, } \\
\text { arteriosclerose e hipertrofia } \\
\text { arterial em locais arteriais } \\
\text { distintos. }\end{array}$ & $\begin{array}{l}\text { Sphygmocor- } \\
\text { AtCor, Sydney, } \\
\text { Austrália) }\end{array}$ & $\begin{array}{l}\text { Avaliação das arterias } \\
\text { carótida e femoral; } \\
\text { Não especificado protocolo } \\
\text { de avaliação. }\end{array}$ \\
\hline $\begin{array}{l}\text { Pieringer et al. } \\
\text { (2012) }\end{array}$ & $\begin{array}{l}\text { Estimar o impacto da AR } \\
\text { sobre o AIx em pacientes } \\
\text { com AR e fatores de } \\
\text { risco cardiovasculares } \\
\text { tradicionais. }\end{array}$ & $\begin{array}{l}\text { Sphygmocor- } \\
\text { AtCor, Sydney, } \\
\text { Austrália) }\end{array}$ & $\begin{array}{l}\text { Avaliação da artéria radial; } \\
\text { Paciente deitado em repouso } \\
\text { por mais de } 5 \text { minutos; } \\
\text { Realizadas a média de duas } \\
\text { medidas. }\end{array}$ \\
\hline $\begin{array}{l}\text { Kim et al. } \\
(2012)\end{array}$ & $\begin{array}{l}\text { Investigar a prevalência de } \\
\text { doença arterial periférica } \\
\text { e identificar fatores que } \\
\text { influenciam a rigidez } \\
\text { arterial em pacientes } \\
\text { coreanos com AR. }\end{array}$ & $\begin{array}{l}\text { (VP-2000, } \\
\text { Colin Co., Ltd., } \\
\text { Komaki, Japan }\end{array}$ & $\begin{array}{l}\text { Avaliação CB e CD; } \\
\text { Paciente em posição supina } \\
\text { e repouso por mais de } 5 \\
\text { minutos; } \\
\text { Realizadas duas medidas } \\
\text { em hemicorpos direito e } \\
\text { esquerdo. }\end{array}$ \\
\hline $\begin{array}{l}\text { Pieringer et al. } \\
(2010)\end{array}$ & $\begin{array}{l}\text { Investigar a rigidez arterial } \\
\text { na arteria braquial medida } \\
\text { pela VOP em mulheres } \\
\text { com AR de longa duração } \\
\text { e comparar os resultados } \\
\text { com controles saudáveis e } \\
\text { pacientes com fatores de } \\
\text { riscos tradicionais sem AR. }\end{array}$ & $\begin{array}{l}\text { Sphygmocor- } \\
\text { AtCor, Sydney, } \\
\text { Austrália) }\end{array}$ & $\begin{array}{l}\text { Avaliação das artérias } \\
\text { carótida e radial; } \\
\text { Utilizada a média de três } \\
\text { medidas; } \\
\text { ECG simultaneamente. }\end{array}$ \\
\hline $\begin{array}{l}\text { Bota et al. } \\
(2016)\end{array}$ & $\begin{array}{l}\text { Avaliar o efeito das } \\
\text { características do HDL sobre } \\
\text { rigidez arterial em pacientes } \\
\text { com AR classificados de } \\
\text { acordo com a atividade da } \\
\text { doença inflamatória. }\end{array}$ & $\begin{array}{l}\text { Complior }{ }^{\circledR} \\
\text { Colson AS, } \\
\text { París, France }\end{array}$ & $\begin{array}{l}\text { Avaliação das artérias } \\
\text { carótida e femoral; } \\
\text { Não especificado protocolo } \\
\text { de avaliação. }\end{array}$ \\
\hline $\begin{array}{l}\text { Magda et al. } \\
\text { (2016) }\end{array}$ & $\begin{array}{l}\text { Avaliar as disfunções } \\
\text { cardíacas subclínicas e } \\
\text { vasculares e os mecanismos } \\
\text { das interações arterio } \\
\text { ventriculares em pacientes } \\
\text { em tratamento para AR vs } \\
\text { sujeitos controles. }\end{array}$ & $\begin{array}{l}\text { Complior }^{\circledR} \\
\text { Colson AS, } \\
\text { Paris, France }\end{array}$ & $\begin{array}{l}\text { Avaliação das artérias } \\
\text { carótida e femoral; } \\
\text { Paciente em repouso por } 15 \\
\text { minutos; } \\
\text { Medidas realizadas somente } \\
\text { em membro superior direito } \\
\text { (MSD). }\end{array}$ \\
\hline
\end{tabular}




\section{Fisi

\begin{tabular}{|c|c|c|c|}
\hline $\begin{array}{l}\text { Autor e ano de } \\
\text { publicação }\end{array}$ & Objetivo & Sistema VOP & Método avaliação VOP \\
\hline $\begin{array}{l}\text { Mangoni et al. } \\
\text { (2017) }\end{array}$ & $\begin{array}{l}\text { Identificar se a } \\
\text { cardioproteção associada } \\
\text { ao MTX envolve alterações } \\
\text { na pressão arteria e ou na } \\
\text { função arterial. }\end{array}$ & $\begin{array}{l}\text { SphygmoCor } \\
\text { AtCor Medical, } \\
\text { Sydney, } \\
\text { Austrália } \\
\\
\text { Mobil-O- } \\
\text { Graph PWA; } \\
\text { IEM, Stolberg, } \\
\text { Alemanha }\end{array}$ & $\begin{array}{l}\text { Avaliação das artérias } \\
\text { carótida braquial; } \\
\text { Não especificado protocolo } \\
\text { de avaliação. }\end{array}$ \\
\hline $\begin{array}{l}\text { Pieringer et al. } \\
(2017)\end{array}$ & $\begin{array}{l}\text { Avaliar a associação entre } \\
\text { dois conceitos, calculadoras } \\
\text { de risco cardiovascular e } \\
\text { parâmetros para avaliação } \\
\text { da função vascular. }\end{array}$ & $\begin{array}{l}\text { SphygmoCor- } \\
\text { AtCor Medical, } \\
\text { Sydney, } \\
\text { Austrália }\end{array}$ & $\begin{array}{l}\text { Realizadas a média de duas } \\
\text { medidas; } \\
\text { Não especificado protocolo } \\
\text { de avaliação. . }\end{array}$ \\
\hline $\begin{array}{l}\text { Mathieu et al. } \\
\text { (2012) }\end{array}$ & $\begin{array}{l}\text { Investigar os efeitos de } 24 \text { e } \\
52 \text { semanas de tratamento } \\
\text { com rituximabe na rigidez } \\
\text { arterial e fatores de risco } \\
\text { cardiovascular. }\end{array}$ & $\begin{array}{l}\text { SphygmoCor- } \\
\text { AtCor Medical, } \\
\text { Sydney, } \\
\text { Austrália }\end{array}$ & $\begin{array}{l}\text { Avaliação das artérias } \\
\text { carótida, femoral e radial; } \\
\text { Membro superior dominante }\end{array}$ \\
\hline
\end{tabular}

Fonte: Elaborado pelas autoras

(clique para voltar ao texto) 